G. Comte, M. Marcelin, eds.

\title{
The O.A.O. Spectronebulagraph and the Kyoto 3D Spectrograph
}

\author{
Hiroshi Ohtani \\ Department of Astronomy, Kyoto University Kyoto 606-01, Japan
}

\begin{abstract}
Spectronebulagraph, an automated observation system of tridimensional spectroscopy with a long slit spectrograph, is now in operation at the $188 \mathrm{~cm}$ telescope of the Okayama Astrophysical Observatory. This system is based on the local area net work of personal computers which control the telescope, the autoguider, the Cassegrain spectrograph and its CCD camera. Effects of variable atmospheric transmission during scans of target objects are removed by performing a set of two orthogonal scans and the data processing.

A new spectrograph is now under development for the telescope. This instrument has multiple modes for tridimensional spectroscopy, i.e., the filter imagery mode, the imaging Fabry-Perot interferometer mode, a slit spectrograph mode for Spectronebulagraph, and the microlens array spectrograph (TIGER) mode. For the last mode, a dual preoptics to get simultaneously the sky background is introduced and achromatization of the microlenses is tried. This project is a part of development of a prototype instrument for the SUBARU telescope.
\end{abstract}

\section{Introduction}

For these several years, our group of Kyoto University has collaborated with the National Astronomical Observatory in development of facilities for tridimensional optical spectroscopic observations. This article is a review of these works.

The first project was the development of Spectronebulagraph (SNG) which is now in operation as a common observatory instrument of the $188 \mathrm{~cm}$ telescope of the Okayama Astrophysical Observatory (OAO) (Kosugi et al. 1994). The system structure and the performance is briefly described below. Some astronomical results obtained with this system are presented in this colloquium by Yoshida et al., Aoki et al. and Kosugi et al..

The next project which is in progress is the development of a new spectrograph with multiple modes for tridimensional spectroscopic observations as a prototype instrument for the SUBARU telescope (Ohtani et al. 1994). The design is shortly presented. 


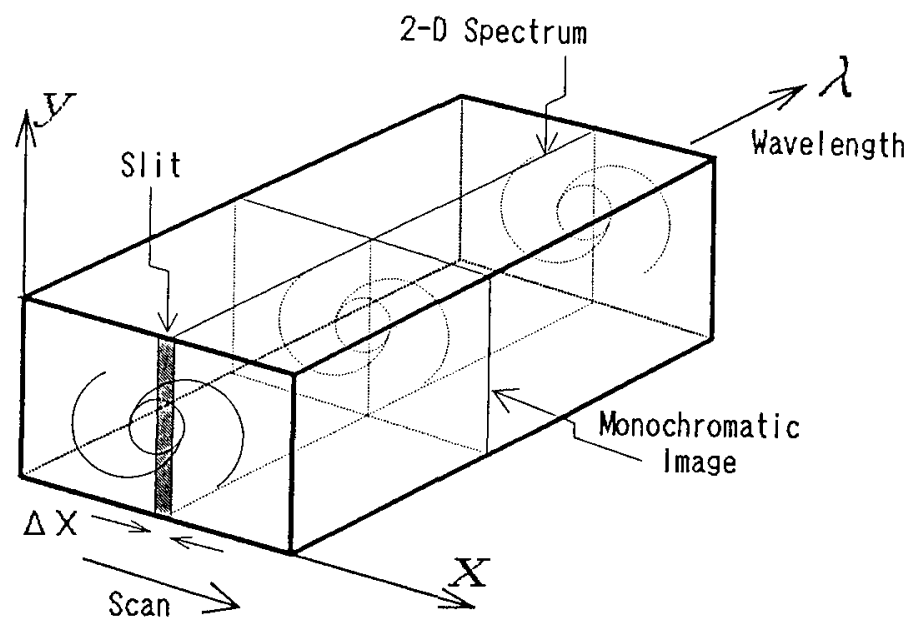

Figure 1. The concept of SNG observations.

\section{The Spectronebulagraph}

One of the most straight forward methods to obtain data cubes of the extended objects is to acquire a series of two dimensional spectra by scanning a target area of the sky with a slit spectrograph (figure 1).

Spectronebulagraph is an automated observation system to perform such a mode of spectroscopy. The system been developed with consideration that the operation is to be so easy that the system can be used as one of the common observatory facilities of the observatory.

This system is based on the local area network of personal computers (PCs) in the telescope dome. In figure 2, the block diagram of the hardware system of SNG is shown.

The system consists of four subsystems, i.e., the driving system of the fine motion of the telescope, the autoguider system, the control system of the Cassegrain spectrograph and the CCD camera system. Each device is controlled by the respective host PC. The spectrograph and the CCD camera share one PC named SNG PC as their hosts. These computers are linked to one another by a network with the server PC.

The concept of the structure of the software system is illustrated in figure 3. On the SNG PC, the SNG observation program runs. The interface between observers and the SNG program is a subprogram named PROC. This consists of a batch of several macroscopic commands named JOBs. Among them, the two essential JOBs are MOVE TELESCOPE and EXPOSURE which run in turn repeatedly in SNG observations. The parameters such as the step size of the telescope movement, the exposure time and so on are input by the observer prior to a scan. Every JOB is an assembly of COMMANDs each of which corresponds to an elementary action of a device, e.g., open/close of the shutter of the CCD camera. 


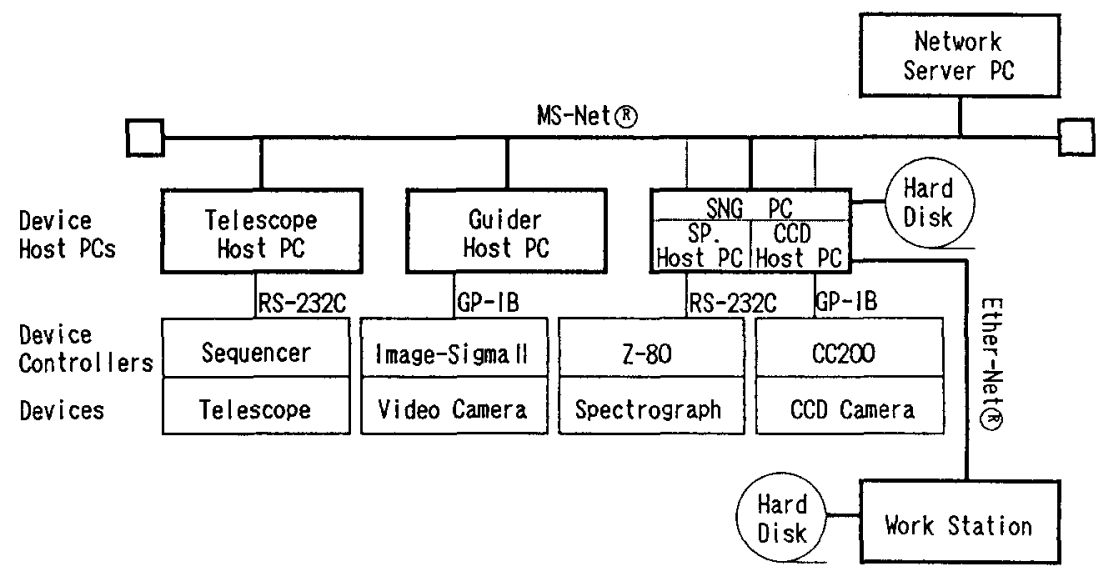

Figure 2. A block diagram of the hardware of the SNG system.

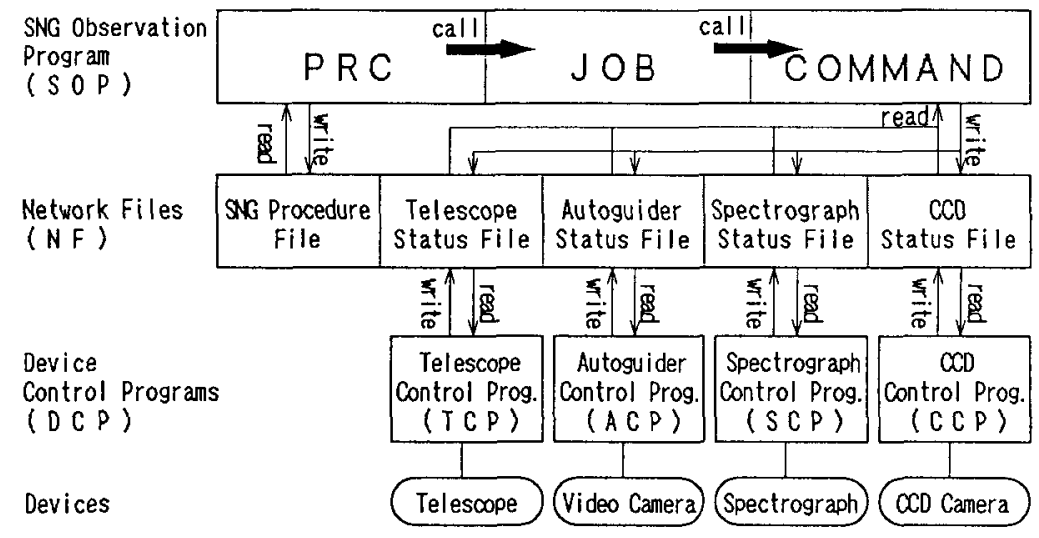

Figure 3. The structure of the software of the SNG system. 
COMMANDs, however, do not access directly to the device hosts. Communications between the observation program and the device control programs are made via a file prepared in the server computer. This file is called as status file since the status of every part of the all devices is registered. There are two kinds of status for one part, the requested status and the current status. These two kinds of status on the file are monitored by the corresponding device control program. The actual action of a COMMAND is to write the requested status in the file. If the requested status is different from the current status, the device control program executes the requested action and then updates the current status file.

The three programs which share the SNG PC do not interact directly each other but through the status file. Thus the status file is a software interface between the observation program and the control programs on the device hosts. In this method, several devices can be controlled diversely to attain SNG observations.

Since a long slit spectrograph is used for the SNG system, an area whose width is as wide as 6 arcmin is scanned in one observation. The maximum wavelength coverage of the data cube is $2400 \mathrm{~A}$ for the lowest spectral dispersion of $4.8 \mathrm{~A} / \mathrm{pixel}$. As the average size of the seeing at the telescope site is 2 arcsec, one step of the scan of object images is usually taken as 1.8 arcsec which is equal to the slit width. The movement of the telescope for the scan is achieved by controlling the autoguider. When a 14th magnitude star is used as the guide star, the geometrical accuracy of the obtained data cube is 0.3 arcsec which much smaller than the step size.

In SNG observations, there are dead times which originate from two major sources. The one is the time required for movement of the telescope. Because of some slow response in the mechanical actions of the fairly old telescope, it takes about as long as 20 sec with a 14th magnitude guide star from a slit position to the next. The other major source of the dead time is the time required to read out and store the CCD data, which is about $15 \mathrm{sec}$. Including the other minor sources, a typical total dead time between successive two exposures is 1 min or less. Therefore, a longer dwell time for exposure at one slit position on the object image is preferred to suppress the fraction of the dead time in a total observation time, while the exposure time is limited at most to $30 \mathrm{~min}$ by the radiation events on the $C C D$.

A similar system to SNG at the AAT, ASPECT (Clark and Wallace 1984), can be operated practically without dead time. In the case of ASPECT, the telescope has a very rapid mechanical response and the photon counting detector which has virtually no dead time in the data acquisition is used. Therefore, the object can be scanned rapidly many times to avoid affection of changes of the sky condition.

In nights with variable transmission due to changes of the sky condition, data cubes obtained by SNG observations of long total observation times suffer from spacial inhomogeneities along the direction of the scan. However, this effect can be successfully removed by performing a set of two scans whose directions are perpendicular each other. In the data processing, each of the $2 \mathrm{D}$ spectra thus obtained is scaled in intensity respectively so that the spectral intensities 
from the two different scans coincide at all points common to the both scans. The scaling factors can be determined by the least-squares method.

\section{The Kyoto 3D Spectrograph}

In principle, the efficiencies of tridimensional spectroscopic observations are same as long as the full area of the same detector is utilized. However, the most favourable method should be selected for the purpose of an observation. Occasionally, an observer needs to apply more than two method for a single object in his one session of observation. Therefore, instruments equipped with multiple functions are often very useful. With this motivation, we started a project to develop a new spectrograph with multiple modes as a prototype instrument for the Subaru.

The instrument can be used in four ways, i.e., in the imager mode with narrow (or wide) band filters, the long-slit spectrograph mode for Spectronebulagraph observations, the Fabry-Perot interferometer imager mode, and the microlens-array (MLA or TIGER (e.g., Courtes et al. 1988)) spectrograph mode. Selection of the mode is made quickly by moving the relevant optical parts of the spectrograph.

The schematic layout of the optical configuration of the spectrograph is illustrated in figure 4. The parameters of the optical elements are also given in the figure. These parameters are determined with consideration of the primary use of the MLA mode at the 188 telescope. One unit is selected among the three at the telescope focal part. The unit consisting of an aperture plate followed by an enlarger lens and a small folding mirror is the preoptics of the MLA spectroscopy mode. According to the selected mode, the large folding mirror within the collimator lens systems is moved. The collimator systems (L4a,b,c + L5Aa,b + L5B) are optically identical for the all modes. The camera lens is used in the telecentric configuration.

Both in the conventional filter imager and the Fabry-Perot imager modes, the object image of the scale $6.1 \mathrm{arcsec} / \mathrm{mm}$ at the telescope focus is projected on the CCD with a magnification 0.27 . The CCD has $1024 \times 1024$ pixels each of which is 12 micron meter square. Two by two binning is applied in observations. The field of view of the imager mode is limited by the detector area and is 4.6 arcmin square. These specifications are tabulated in table 2. The same spacial scaling applies along the slit of the conventional spectrograph mode.

Two scanning Fabry-Perot etalons of the model ET50 of Queensgate Instruments are prepared for this spectrograph. One etalon $(R=330)$ is a tunable filter which is to be used for imaging in various emission line imaging of active galaies of different redshifts. The other etalon has a much higher spectral resolution ( $R=10000$ ) This will be applied to velocity field measurements of galaxies. Some parameters of the Fabry-Perot imager mode are given also in table 1.

In the MLA spectrograph mode, the image at the telescope focal plane is projected on the MLA with a magnification 15 times. With this magnification, only a very narrow filed of view is observable. Therefore, the nearby sky cannot be observed simultaneously with the object with one channel optical system. Considering the importance of the subtraction of the sky background spectra 


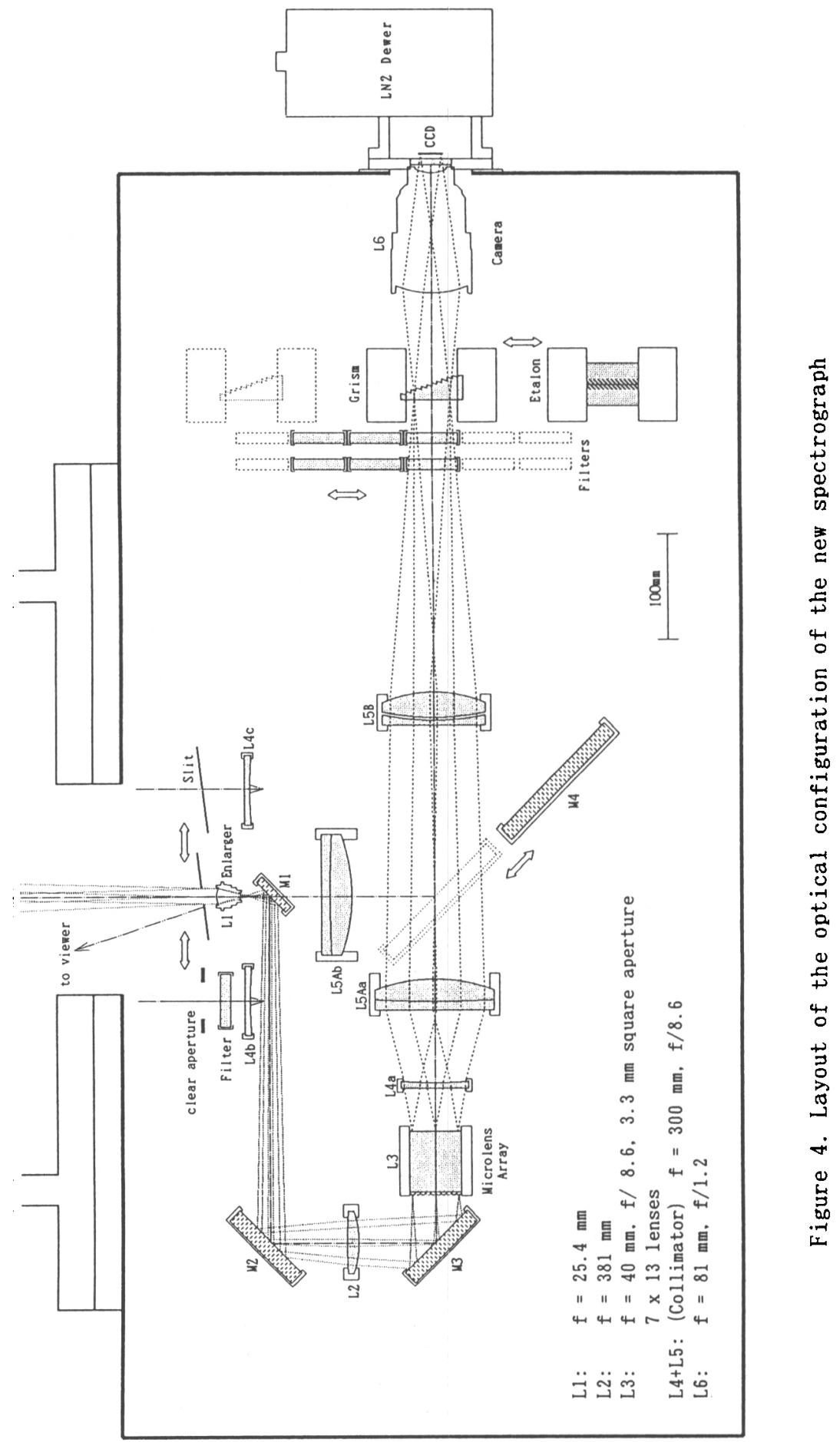


Table 1. Filter and Fabry-Perot imager modes

\begin{tabular}{cccc}
\multirow{2}{*}{$\begin{array}{c}\text { Telescope } \\
(\mathrm{f} / 18)\end{array}$} & $\begin{array}{c}\text { Spatial } \\
\text { resolution }\end{array}$ & \multicolumn{2}{c}{ Field of view (arcmin) } \\
\cline { 3 - 4 }$\left(\right.$ arcsec/pix $\left.{ }^{*}\right)$ & Total(square) & Monochromatic(diam) \# \\
\hline $8.2 \mathrm{~m}$ & 0.12 & 1.0 & 0.34 \\
$1.88 \mathrm{~m}$ & 0.54 & 4.6 & 1.5 \\
$0.6 \mathrm{~m}$ & 1.9 & 14.5 & 4.7 \\
\hline
\end{tabular}

* $24 \mu \mathrm{m}$ square

\# Within this area, differences of the central wavelengths are less

than one tenth of the FWHM of the etalon with $R=330$

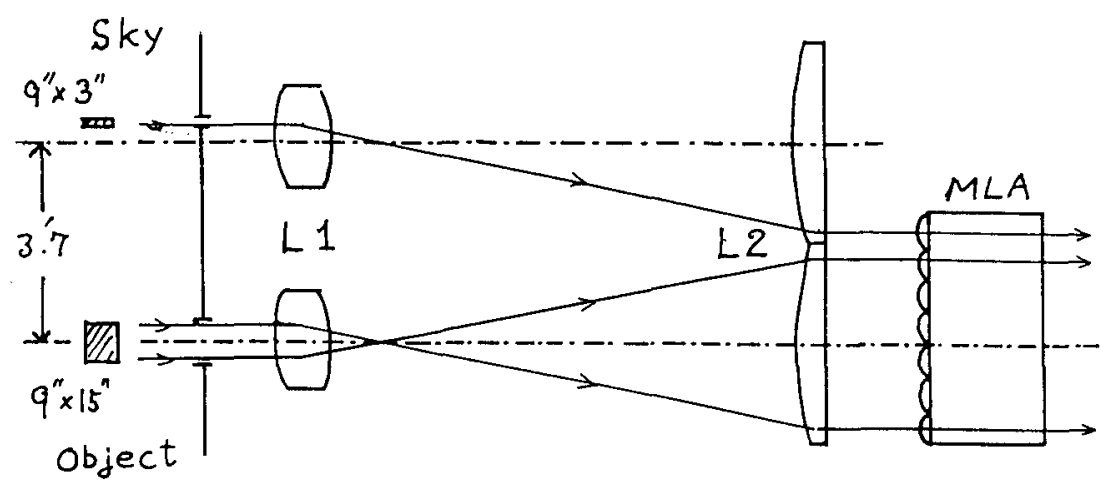

Figure 5. The preoptics of the MLA spectrograph mode.

taken simultaneously, we have introduce a preoptics system of dual channels whose principle is explained by the schematical figure 5 .

The main channel is used to obtain the spectra of the target field, while the other is for the nearby sky. An area apart by as far as 3.7 arcmin from the field center of the object channel is acquired by the sky channel.

The MLA consists of ninety one small thin planoconvex lenses cemented on one side of a thick plane parallel glass plate. The aperture of each lens is 3.3 $\mathrm{mm}$ square. By adopting BK7 and SF2 for the planoconvex lenses and the plane parallel plate, respectively, the chromatic aberrations are appreciably reduced.

After the microlens array, an array of the pupils with diameter 154 micron meter are made. These pupils are dispersed into an array of spectra on the CCD. The parameters of the MLA mode is given in table 2.

This spectrograph may have opportunity to be used at other telescopes, the $60 \mathrm{~cm}$ reflector of Kyoto University and the SUBARU. In tables 1 and 2, the parameters in these cases are also tabulated.

Acknowledgments. The author is indebted to the collaborators, G. Kosugi, M. Yoshida, T. Sasaki, Y. Shimizu, H. Koyano, M. Sasaki, K. Aoki, T. 
Table 2. Microlens array mode

$7 \times 11$ spectra are for the object area and $2 \times 7$ for the sky.

\begin{tabular}{ccc}
\hline $\begin{array}{c}\text { Telescope } \\
(\mathrm{f} / 18)\end{array}$ & $\begin{array}{c}\text { Spacial resolution } \\
\text { (arcsec sq) }\end{array}$ & $\begin{array}{c}\text { Field of view* } \\
\text { (arcsec } \times \text { arcsec) }\end{array}$ \\
\hline $8.2 \mathrm{~m}$ & 0.3 & $2.1 \times 3.4$ \\
$1.88 \mathrm{~m}$ & 1.3 & $9.4 \times 14.8$ \\
$0.6 \mathrm{~m}$ & 4.1 & $29 \times 45$ \\
\hline
\end{tabular}

Ishigaki, H. Maemura, and J. Noumaru. He also thanks IAU for the financial support for the travel.

\section{References}

Clark, D. and Wallace, P., 1984, QJRAS, 25, 114.

Courtes, G., Georgelin, Y., Bacon, R., Monnet, G. and Boulesteix, J., 1988, in Instrumentation for ground based optical astronomy, ed. L. B. Robinson, Springer-Verlag, New York, p267.

Kosugi. G., Ohtani, H., Yoshida, M., Sasaki, T., Shimizu, Y., Koyano, H., Aoki, K., Sasaki, M., 1994, to be submitted to PASP.

Ohtani, H., Sasaki, M., Aoki, K., Takano, E., Kiyohara, M., 1994, in the Proceedings of Instrumentation in Astronomy VIII, in press. 